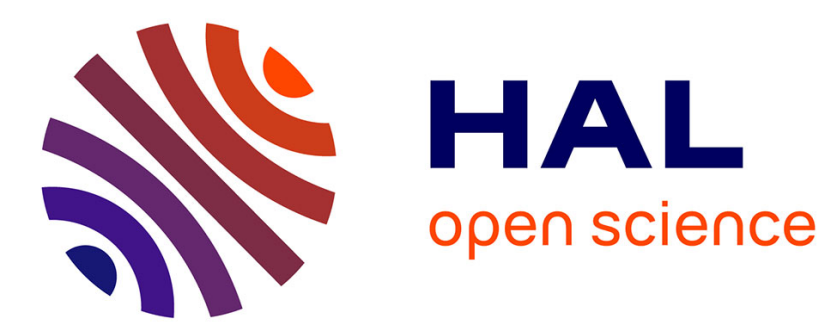

\title{
La consommation des jeux de hasard: un exemple d'application de la complexité à un objet sociologique
}

Audrey Valin

\section{To cite this version:}

Audrey Valin. La consommation des jeux de hasard: un exemple d'application de la complexité à un objet sociologique. Sociétés, 2007, p. 65-80. hal-00484437

\section{HAL Id: hal-00484437 \\ https://hal.science/hal-00484437}

Submitted on 18 May 2010

HAL is a multi-disciplinary open access archive for the deposit and dissemination of scientific research documents, whether they are published or not. The documents may come from teaching and research institutions in France or abroad, or from public or private research centers.
L'archive ouverte pluridisciplinaire HAL, est destinée au dépôt et à la diffusion de documents scientifiques de niveau recherche, publiés ou non, émanant des établissements d'enseignement et de recherche français ou étrangers, des laboratoires publics ou privés. 


\section{LA CONSOMMATION DES JEUX DE HASARD : UN EXEMPLE D'APPLICATION DE LA COMPLEXITE À UN OBJET SOCIOLOGIQUE}

Audrey Valin

\section{Introduction :}

L'exemple des jeux de hasard présenté ici brièvement est extrait d'un travail mené durant deux années dans le cadre d'un Master de sociologie à l'Université François Rabelais de Tours ${ }^{1}$. Cet objet a été choisi comme étant une première piste d'approche de la notion d'aléa que nous souhaitons aborder de façon sociologique. La pensée complexe qu'offre Edgar Morin est fondamentale en ce sens qu'elle réhabilite le hasard et permet de l'intégrer à la réflexion. La difficulté reste de définir cette notion source de tant d'incertitude : "Ainsi, d'une part, nous devons constater que le désordre et le hasard sont présents dans l'univers et actifs dans son évolution, d'autre part, nous ne pouvons résoudre l'incertitude qu'apportent les notions de désordre et de hasard; le hasard lui-même n'est pas certain d'être hasard. L'incertitude demeure, y compris en ce qui concerne la nature de l'incertitude que nous apporte le hasard $»^{2}$. En étudiant les jeux de hasard, nous tentons de comprendre une activité sociale qui met en scène l'aléa. En posant le problème en terme d'action, nous souhaitons appréhender les stratégies que les individus mettent en place face au hasard, car « l'action est stratégie [et] la stratégie ne se borne pas à lutter contre le hasard, elle essaie aussi de l'utiliser $»^{3}$.

Communément, la sociologie est représentée par le paradigme atomistique ou individualiste méthodologique (attribué à Max Weber et Georg Simmel notamment) et le paradigme holistique ou déterministe (historiquement représenté par Emile Durkheim ou encore Marcel Mauss). Cependant, il faut se méfier des cloisonnements par trop tranchés sur les positions théoriques des auteurs (à ce propos, voir notamment la préface de Michel Maffesoli à la réédition de l'ouvrage d'Emile Durkheim Les Formes élémentaires de la vie religieuse ${ }^{4}$ ). Le premier paradigme veut comprendre le tout à partir uniquement des qualités des parties, tandis que le second néglige les parties pour comprendre le tout. Néanmoins c'est là qu'ils se rejoignent, puisque chacun, à sa façon, présente un modèle linéaire pour expliquer un phénomène. Or, aujourd'hui, Edgar Morin propose un type d'explications différent : « en mouvement, circulaire », où l'on va des parties au tout, du tout aux parties $»^{5}$, suivant les principes hologrammatique 6 et de récursion organisationnelle selon lesquels le tout (la société) est produit pas les interactions entre les parties (les individus), en même temps que ces parties sont coproduites par ce tout organisateur qui rétroagit sur elles (par l'éducation, la culture et le langage $)^{7}$. À cela doit être ajouté le principe dialogique qui « nous permet de maintenir la dualité au sein de l'unité [en associant] deux termes à la fois complémentaires et antagonistes $\gg{ }^{8}$. Inséparables les uns des autres, ces trois principes permettent de penser ce que Morin nomme la complexité et qui suit l'idée de son précurseur Gaston Bachelard selon laquelle il n'y a rien de simple dans la nature, il n'y a que du simplifié. Mais il est difficile de parvenir à la pensée complexe car elle implique de se débarrasser d'une "raison close qui

\footnotetext{
1. L'intégralité du mémoire est disponible à Maison des Sciences de l'Homme de Tours, sous le titre $\mathrm{La}$ consommation des jeux de hasard, Mémoire de Master, 2007.

${ }^{2}$. MORIN E., Science avec conscience, Paris, Fayard, 1982, p. 166.

${ }^{3}$. MORIN E., Introduction à la pensée complexe, Paris, Seuil, 2005, p. 106.

${ }^{4}$. DURKHEIM E., Les Formes élémentaires de la vie religieuse, Paris, Librairie générale française, 1991 (1912).

${ }^{5}$. MORIN E., Science avec conscience, Paris, Fayard, 1982.

${ }^{6}$. Ibid., p. 168.

7. MORIN E., Introduction à la pensée complexe, Paris, Seuil, 2005, pp. 98-99.

${ }^{8}$. Ibid., p. 99.
} 
rejette comme inassimilable des pans énormes de réalité $)^{9}$ en restant enfermée dans le carcan, notamment de la binarité. Or, cette dernière est le fruit d'un rationalisme qui s'est érigé comme éthique et qui affirme l'accord parfait entre la cohérence et la réalité de l'univers. C'est pourquoi Edgar Morin, qui voit la raison comme « une méthode de connaissance fondée sur le calcul et la logique, employée pour résoudre des problèmes posés à l'esprit, en fonction des données caractérisant une situation ou un phénomène ${ }^{10}$ en propose une vision complexe, c'est-à-dire ouverte, qui « ne conçoit plus en opposition absolue, mais en opposition relative, c'est-à-dire aussi en complémentarité, en communications, en échanges des termes jusqu'alors antinomiques: intelligence et affectivité ; raison et déraison. Homo n'est pas seulement sapiens, mais sapiens/demens ${ }^{11}$. Le défi est excitant à relever et nous adhérons d'autant plus à ce mode de pensée que notre objet touche directement au premier chemin que propose l'auteur pour penser la complexité : « l'irréductibilité du hasard ou du désordre » ${ }^{12}$. D'une manière générale et dans une démarche globale, c'est donc cette façon de penser que nous tâchons d'adopter au cours de nos recherches. En outre, c'est bien en tant qu'activité de la « vie quotidienne » que nous étudions le jeu de hasard, suivant l'idée de Claude Rivière selon laquelle : «c'est dans l'apparemment anodin de la vie quotidienne que se révèlent les modèles culturels, aspects fondamentaux de la vie, qui orientent les comportements des membres d'un groupe $»^{13}$.

\section{Le joueur en acte}

\subsection{L'objet des jeux de hasard}

L'analyse du hasard est étymologiquement liée au jeu; le mot est emprunté aux Arabes vers le XII ${ }^{\mathrm{eme}}$ siècle et vient de azzahr qui était un jeu de dés. Actuellement, le dictionnaire Le Robert $^{14}$ nous offre une définition du jeu qui est riche d'enseignements, notamment en ce qui concerne les préjugés dont il pâtit. Selon l'emploi du mot, les définitions sont multiples (l'ouvrage en présente dix-huit); nous présentons les deux premières :

1) «Se livrer au jeu. $\Rightarrow$ s'amuser. Écoliers qui jouent pendant la récréation $[. .]$.

2) « Pratiquer les jeux d'argent. Il boit et il joue »

Deux idées reçues du sens courant sont exprimées. La première associe le jeu à l'enfance sous le prétexte du temps libre : le jeu n'est donc pas l'affaire des adultes qui mettent le temps dont ils disposent à des activités moins futiles. La deuxième définition aborde une catégorie de jeu bien particulière : les jeux d'argent. Ceux-là sont exclusivement des jeux d'adultes, ne seraitce que pour leur vocation lucrative. Et là intervient un autre préjugé quand Le Robert illustre la pratique du jeu d'argent par « Il boit et il joue » : le jeu d'argent est associé à la boisson et déjà se dressent les images du casino tel que Fedor Dostoïevski le décrit à travers les yeux d'Alexis Ivanovitch : « Au premier abord, tout me parut sale, moralement sale et abjecte. Je ne veux pas parler de ces visages avides et inquiets [...]. Ce qui me déplaisait le plus au premier coup d'œil dans toute cette racaille, c'était la gravité, le sérieux, le respect même avec lequel tous ces gens entouraient les tables ${ }^{15}$. C'est évidemment de valeurs morales qu'il est question, à l'instar des critiques antérieures que nous avons exposées précédemment. Nous ne

\footnotetext{
9 Ibid., p. 155.

${ }^{10}$. Ibid., p. 145

${ }^{11}$. Ibid., p. 156

12. Ibid., p. 165

${ }^{13}$. RIVIERE C., «Pour une théorie du quotidien ritualisé », Ethnologie française, n²6, Paris, Armand Colin, 1996, p. 231.

14 . Dictionnaire Le Robert, Paris, Nathan, 1991.

${ }^{15}$. DOSTOÏEVSKI F., Le Joueur, Paris, Gallimard, 1977 (1865), p. 31.
} 
ferons pas ici une analyse des préjugés, comment ils fonctionnent et pourquoi nous les mettons en place. Le but est seulement de présenter les deux aspects qui sont le plus souvent évoqués par le sens commun à propos du jeu : enfant puis argent. Mais le jeu ne concerne pas que les enfants, et les jeux d'argent ne représentent qu'une catégorie parmi d'autres. Alors pourquoi parle-t-on de jeu pour des pratiques si différentes les unes des autres? Les jeux enfantins sont reconnus utiles pour leurs qualités éducatives, d'autres jeux sont jugés inutiles et d'autres, tels que les jeux de grattage, ne sont même pas considérés comme des jeux par la plupart des individus qui n'y voient aucun amusement mais le seul appât du gain. Pas de stratégie dans ces jeux, pas de réflexion, l'argent omniprésent tout au long de l'activité, etc. : ces jeux ne sont pas des jeux. Quelle drôle de formule... Mais pourquoi, alors, les appelle-t-on jeux ? Et pourquoi ont-ils tant de succès ? Au premier abord, en effet, ces tickets à gratter et ces grilles de loterie sont des produits commerciaux. Il ne s'agit pas de réfuter ce constat, c'est bien l'une des dimensions de l'objet. Cependant, nous proposons de ne pas s'en tenir à ce seul aspect.

\subsection{L'" homo ludens" pour comprendre l'action de jouer}

Il s'agit de considérer les jeux de «hasard pur» de la FDJ sous l'angle des trois facteurs qu'ils recèlent directement: l'argent, le hasard et le ludisme. Le but est de comprendre l'action particulière de la pratique des jeux de hasard et de l'expliquer au sens qu'en donne Max Weber dans sa définition de la «compréhension explicative » ${ }^{16}:$ «nous "comprenons", parce que nous saisissons la motivation [motivationsmässig], le sens qu'une personne a associé à la proposition [...] qu'elle prononce ou qu'elle a écrite, à cet instant précis et dans ce contexte [...]. Dans tous les cas, nous avons affaire à des ensembles significatifs [Sinnzusammenhänge] compréhensibles, et nous considérons leur compréhension comme une explication [Erklären] du déroulement effectif de l'activité. Pour une science qui s'occupe du sens de l'activité, "expliquer" signifie par conséquent la même chose qu'appréhender l'ensemble significatif ${ }^{17}$. Nous cherchons à appréhender le sens visé, c'est-à-dire «le sens subjectif du devenir [Geschehen] [car dans] tous les cas, "comprendre" signifie saisir par interprétation le sens ou l'ensemble significatif visé $[\ldots] »{ }^{18}$. Ainsi, les motifs donnés à l'action sont indispensables pour la saisir, en ce sens qu'ils constituent déjà eux-mêmes " un ensemble significatif qui semble constituer aux yeux de l'agent ou de l'observateur la "raison" significative d'un comportement ${ }^{19}$. Dans ce cadre, nous voulons identifier les motifs qui sont actifs et déterminants dans l'action de jouer à des jeux de hasard. C'est donc l'acte de jouer qui est le véritable questionnement. Et par jouer, nous entendons l'action de jouer, dans ce sens qu'il s'agirait d'une action rationnelle dans une activité irrationnelle ${ }^{20}$. D'après Edgar Morin : «La raison froide n'existe pas. Même le mathématicien a des émotions. "L'homo sapiens" est une illusion. Même nos pensées les plus rationnelles sont mêlées d'émotions. [...] Nous sommes tous des êtres de raison et de "jeu". Disons donc aussi : "l'homo ludens" car le jeu n'est pas limité à l'enfance. Il tient une place énorme dans la vie, et même dans l'économie ! ${ }^{21}$. Pour l'auteur, l'instabilité est caractéristique de l'homme; il est désorganisé notamment parce qu'il est rationnel et irrationnel à la fois : "Ainsi, l'être humain ne vit pas que de rationalité et de technique [...] Partout, une activité technique, pratique, intellectuelle,

\footnotetext{
${ }^{16}$. WEBER M., « Les Catégories de la sociologie», Economie et société, Paris, PLON, 1995 (1922), Tome 1, p. 34.

${ }^{17}$. Ibid.

${ }^{18}$. Ibid., p. 35.

19 . Ibid., p 38.

${ }^{20}$. La distinction entre les deux termes est éclairée dans le point suivant.

${ }^{21}$. MORIN E., La Complexité humaine, Paris, Flammarion, 1994.
} 
témoigne de l'intelligence empirico-rationnelle ; [...] en même temps [...] les activités de jeu, de fête, de rite ne sont pas de simples détentes pour se remettre à la vie pratique ou au travail, les croyances aux dieux et aux idées ne peuvent être réduites à des illusions ou superstitions : elles ont des racines qui plongent dans les profondeurs anthropologiques; elles concernent l'être humain dans sa nature même. [...] Nous sommes des êtres infantiles, névrotiques, délirants, tout en étant aussi rationnels. Tout cela constitue l'étoffe proprement humaine. [...] L'être humain est un être raisonnable et déraisonnable, capable de mesure et de démesure ; sujet d'une affectivité intense et instable, il [...] sait aussi connaître objectivement ; c'est un être sérieux et calculateur, mais aussi anxieux, angoissé, jouisseur, ivre, extatique ; c'est un être [...] envahi par l'imaginaire et qui peut reconnaître le réel [...] Il se nourrit de connaissances vérifiées, mais aussi d'illusions et de chimères $»^{22}$. Rationnel et irrationnel ne peuvent donc pas être séparés quand l'on traite de l'être humain; il s'agit de forces complémentaires qui interagissent ensemble en quelque sorte. D'où la nécessité de ne pas raisonner de façon binaire en séparant distinctement les parties d'un même tout. Mais il est difficile de se détacher de ce mode de pensée qui accompagne toujours nos réflexions en tout premier lieu. Tout comme il est difficile de rompre avec notre idéologie de connaissance qui, elle, nous amène à penser le jeu en termes d'utilité. En y appliquant inévitablement le schéma binaire, nous obtenons à propos du jeu la disjonction utilité / inutilité. Utile, il est un acte motivé par un but et des forces matérielles (qui peuvent également comprendre des forces affectives mais restant dans le domaine de la sociabilité). Encore, le jeu est stratégique, il est une relation entre plusieurs individus distincts. Futile, il est un vagabondage, non stratégique, il obéit à des forces immatérielles telles que des croyances, des intuitions, en un mot, de l'abstrait. Autant d'éléments impalpables et donc difficiles à approcher. C'est le contexte même de la relation qui fait appel à des notions telles que celle de hasard. Binaire, la perspective n'est pas erronée, là n'est pas le propos et il convient de la prendre en compte et de s'en inspirer. Cependant, nous préférons adopter le point de vue d'Edgar Morin qui explique que c'est la «relation » qui est au fondement de l'être humain. Or, la relation suppose qu'il est impossible de considérer le biologique (le naturel) comme séparé du social. L'homme est, en quelque sorte, «biologico-social », en tout cas biologique et social à la fois dans une relation permanente. Et c'est bien cela qu'il nous faut prendre en compte : à partir du moment où le jeu est un phénomène visible dans la société, c'est qu'il est également du domaine de la nature de l'homme. C'est ce qu'Edgar Morin appelle «l'homo ludens », c'està-dire le caractère joueur de l'homme et que nous appellerons le ludisme. Mais ce qui est important, c'est de considérer que ce caractère n'est pas isolé des autres natures de l'homme. Ainsi, l'être humain est «complexe et porte en lui de façon bipolarisée les caractères antagonistes : sapiens et demens (rationnel et délirant); faber et ludens (travailleur et joueur); empiricus et imaginarius (empirique et imaginaire); economicus et consumans (économe et dilapidateur); prosaicus et poeticus (prosaïque et poétique). L'homme de la rationalité est aussi celui de l'affectivité du mythe et du délire (demens). L'homme du travail est aussi l'homme du jeu (ludens). L'homme empirique est aussi l'homme imaginaire (imaginarius). L'homme de l'économie est aussi celui de la "consumation" (consumans). L'homme prosaïque est aussi celui de la poésie $[. ..] »^{23}$. Dans la pratique du jeu, le comportement de l'homme révèlerait son inconstance, notamment à travers le rapport qu'il entretient avec le hasard. Nous partons de l'idée suivante : le hasard présente ceci d'irrationnel qu'il n'existe pas. Tout comme la mort, il est une angoisse primaire ${ }^{24}$ que l'homme sublime en l'entourant de représentations, d'images qui lui permettent de donner du sens. Par l'imagination, l'homme

\footnotetext{
22. MORIN E., Les Sept savoirs nécessaires à l'éducation du futur, Paris, Seuil, 2000, pp. 20-30.

${ }^{23}$. Ibid., p. 30.

24. $C f$. LEGROS P., MONNEYRON F., RENARD J-B. et TACUSSEL P., Sociologie de l'imaginaire, Paris, Armand Colin, 2006, pp. 113-116.
} 
parvient à se représenter le hasard et finit surtout par se créer une possible emprise sur lui. Mais il ne s'agit là que d'une tentative de rationaliser l'irrationnel. Alors, par la pratique du jeu, l'homme laisserait s'exprimer une partie irrationnelle de lui-même, sa nature ludique. Son angoisse serait comme matérialisée et l'individu deviendrait acteur de quelque chose qui, par nature justement, le dépasse. Les jeux de hasard seraient le paroxysme de ce rapport en ce sens qu'ils mettent à jour la façon dont l'homme s'en remet totalement au hasard par son activité tandis que ses actions visent à rationaliser l'aléa surnaturel. L'action concernerait les pratiques, les représentations, les comportements qui peuvent se manifester socialement et donc se rendre visibles à l'observateur. Dire que l'action est rationnelle signifie qu'elle apparaît comme tel au regard de la société, alors qu'elle serait mêlée d'irrationnel. Mais cette dernière dimension serait effacée, niée par la pratique parce que mal accueillie par la société, ou alors à condition d'être cadré de sorte à justifier son existence, comme par un processus de bonne conscience. En quelque sorte, on pourrait dire que la société balise une activité irrationnelle pour la rendre socialement acceptable. Le processus consisterait alors à faire paraître l'activité comme rationnelle. L'activité ainsi rationalisée serait l'action.

\subsection{Le symbolisme pour comprendre l'activité}

Afin de percevoir la distinction que nous établissons entre l'activité et l'action, il faut prendre en compte la dimension symbolique telle que Jean Baudrillard la suggère par exemple à l'occasion d'un réflexion sur les objets : "notre civilisation technique tente de compenser l'effacement de la relation symbolique liée au gestuel traditionnel de travail, de surseoir à l'obscénité et au vide symbolique de notre puissance par l'investissement dans les objets, agents intermédiaires d'une mythologie quotidienne qui absorbe l'angoisse du temps et de la mort. S'il advient que les objets se dérobent éventuellement à notre contrôle pratique, "ils n'échappent jamais à l'imaginaire. Les modes de l'imaginaire suivent les modes de l'évolution technologique" $\|^{25}$. L'auteur invite à prendre en compte une dimension symbolique qui investit les objets et qui n'est pas une structure ou une instance, encore moins un concept ou une catégorie, mais un rapport social qui « met fin au réel $»^{26}$ et, du même coup à l'opposition entre le réel et l'imaginaire. C'est d'ailleurs pour cette raison que la sociologie de l'imaginaire ne peut pas être « un champ spécifique de la sociologie, défini par un objet, comme le sont la sociologie urbaine, du travail, de la religion, de l'éducation, etc. Elle est un point de vue sur le social : elle s'intéresse à la dimension imaginaire de toutes les activités humaines $»^{27}$. Dans ce cadre, toute action humaine est investie d'imaginaire en même temps qu'elle répond aux codes sociaux de la rationalité. Les deux termes ne sont pas antinomiques et, en fait, la question de l'imaginaire dépasse l'opposition entre rationalité et irrationalité. C'est ce que nous fait comprendre Cornelius Castoriadis en traitant de ce qu'il nomme l'institution imaginaire de la société (et qui est aussi le titre de l'ouvrage qui l'a fait connaître en 1975): "Tout ce qui se présente à nous, dans le monde social-historique, est indissociablement tissé au symbolique $»^{28}$. Pour lui, la société fabrique son symbolisme, qui est accroché à la fois à la nature et à l'histoire, et participe enfin au rationnel. Ces images et visions utilisent les segments et les modèles rationnels du donné; cependant, elles les ordonnent à des significations qui, en tant que telles, ne relèvent pas du rationnel (ou même d'un irrationnel), mais de l'imaginaire. L'activité relèverait du domaine de l'imaginaire, en ce sens que «ce sont des représentations de "quelque chose" qui orientent les activités

\footnotetext{
25 . BAUDRILLARD J., Le Système des objets, Paris, Denoël-Gonthier, 1968, p. 142.

${ }^{26}$. BAUDRILLARD J., L'Echange symbolique et la mort, Paris, Gallimard, 1976, p. 204.

27. LEGROS P., MONNEYRON F., RENARD J-B. et TACUSSEL P., Sociologie de l'imaginaire, Paris, Armand Colin, 2006, p. 1.

${ }^{28}$. CASTORIADIS C., L'Institution imaginaire de la société, Paris, Seuil, 1999, p. 174.
} 
humaines $»^{29}$ dans une dynamique dont dérive l'action sociale ${ }^{30}$. Ainsi, l'activité est la dynamique de l'action et l'action est l'activité en train de se dérouler. L'action est du domaine du social, elle est en quelque sorte la concrétisation sociale de l'activité. C'est alors au niveau de l'activité que se trouverait le ludisme qui serait concrétisé, matérialisé dans l'action. Nous considérons le jeu comme une action rationnelle dans une activité irrationnelle, au sein de la relation, source de tout acte. Il faut alors entendre la "relation » telle que la définit Edgar Morin $^{31}$ et qui nous permet de sortir des schémas traditionnels de la sociologie.

\section{Le joueur : un consommateur passif, en théorie}

\section{2-1 Discours sur la " culture populaire " ${ }^{32}$}

Les jeux de hasard pâtissent d'une mauvaise réputation qui tient notamment au fait qu'ils contrarient les valeurs de mérite, d'effort ou encore de travail, que promulgue une société historiquement industrielle. Ainsi les notions de plaisir, de désir, de passion ou encore d'émotion ne font pas partie du vocabulaire de l'éthique rationnelle dominante. C'est pourquoi la paresse du joueur qui tente de gagner de l'argent facilement et sans effort est au pire condamnée sans exergue et au mieux attribuée par condescendance aux plus "faibles » individus de la population, aux plus «fragiles », c'est-à-dire communément les " classes populaires ». Très peu définie mais beaucoup employée, cette culture désigne des sphères élargies de la population : elle peut renvoyer étymologiquement à la masse, c'est-à-dire au plus grand nombre et est assimilée ainsi à la société de masse. La distinction entre les deux expressions «culture de masse » et «culture populaire » serait sociale : la culture de masse regroupe les différentes pratiques d'un groupe nombreux, socialement hétérogène : Edgar Morin la définit comme "produite selon les normes massives de la fabrication individuelle, répandue par des techniques de diffusion massive (qu'un étrange néologisme anglo-latin, appelle mass-média), s'adressant à une masse, c'est-à-dire un gigantesque agglomérat d'individus saisi en deçà et au-delà des structures internes de la société (classe, famille...) » ${ }^{33}$. La culture populaire renverrait plutôt à la culture de la classe dite populaire, définie en matière de capital économique faible et de domination par les détenteurs des pouvoirs économiques. Si longtemps cette classe sociale put être délimitée selon des critères précis, comme le revenu, l'évolution de la société a entraîné une reformulation des classes sociales. Ainsi Richard Hoggart, en 1970 dans La Culture du pauvre, montrait la difficulté à définir cette classe sociale. Dans une partie de son étude intitulée « la classe populaire », il dresse une esquisse de définition : «il n'est pas facile de définir précisément les "classes populaires" au sens où mon étude les prend pour objet... Sauf erreur de ma part, les attitudes que je décris dans la première partie de l'ouvrage sont suffisamment répandues au-delà de la classe ouvrière proprement dite, dans l'ensemble des couches sociales qui constituent ce qu'on peut appeler les "classes populaires" pour que l'analyse garde une portée générale. En particulier, mainte attitude que je qualifie globalement de "populaire" pourrait aussi bien être attribuée aux couches inférieures de la petite bourgeoisie. Je ne vois pas comment on pourrait éviter ce genre d'approximation $»^{34}$. Même si Dominique Wolton se range du côté de Richard Hoggart en postulant que, désormais, il conviendrait mieux de parler de «culture moyenne ${ }^{35}$, le

\footnotetext{
${ }^{29}$. LEGROS P., MONNEYRON F., RENARD J-B. et TACUSSEL P., Sociologie de l'imaginaire, Paris, Armand Colin, 2006, p. 105.

${ }^{30}$. Ibid.

31. MORIN E., Science avec conscience, Paris, Fayard, 1982.

${ }_{32}$. Le discours dominant sur les classes populaires est traité dans le mémoire pp. 69-77.

33. MORIN E., L'Esprit du temps. I Névrose, Paris, Grasset, 1962, p. 15.

${ }^{34}$. HOGGART R., La Culture du pauvre, Paris, Minuit, 1970, p. 44.

${ }^{35}$. WOLTON D., Eloge du grand public. Une théorie critique de la télévision, Paris, Flammarion, 1990, p. 198.
} 
terme culture populaire est encore très usité. Cette culture est un élément de la culture de masse et les jeux de grattage demeurent un objet de la culture populaire dans les représentations collectives, malgré les chiffres de la FDJ qui montrent que ses clients viennent, presque uniformément, de toutes les catégories sociales confondues. D'abord, la simplicité du fonctionnement de ces jeux participe à leur dévalorisation. Mais c'est surtout la dimension matérielle de ces jeux qui suscite le débat sur la culture de masse. En effet, c'est le support (ticket à gratter ou grille de loterie) qui révèle la dimension commerciale du jeu qui, elle, évince, a priori, une quelconque valeur ludique. En considérant notamment le marketing dont ils sont l'objet, ces bouts de papiers apparaissent comme des biens uniquement destinés à la consommation de masse caractéristique de la société du même nom. Dans ce cadre, l'École critique postule une culture homogénéisée, homogénéisante et aliénante pour le consommateur et considère que le récepteur est passif. Concernant les jeux de hasard que nous étudions, leur nature marchande oblige à considérer un aspect déterminant dans l'action de jouer. Cependant, considérant la "complexité » de l'homme telle que la définit Edgar Morin, nous devons prendre en compte la part active du joueur tout autant que sa passivité. Une ouverture vers un récepteur actif est apportée par Jürgen Habermas qui propose à l'individu une solution pour résister à la domination ; cela passe par la communication et par l'espace public. Ensuite, les « Cultural Studies » et les « Uses and Gratifications » font parties des avancées théoriques qui contribuent à réhabiliter la part active de l'homme dans son action. Richard Hoggart, par exemple, à propos des productions massives de l'industrie culturelles, dit que «le volume [...] est tel que le chercheur est toujours inconsciemment incliné (en quelque sorte par l'effet de poids) à surestimer [son] influence sur les classes populaires. Il ne faut jamais oublier que ces influences culturelles n'ont qu'une action fort lente sur la transformation des attitudes et qu'elles sont souvent neutralisées par des forces plus anciennes. Les gens du peuple ne mènent pas une vie aussi pauvre qu'une lecture, même approfondie, de leur littérature, le donnerait à penser. Il n'est pas aisé de démontrer rigoureusement une telle affirmation, mais un contact continu avec la vie des classes populaires suffit à en faire prendre conscience $»^{36}$. C'est dans ce souci de coller à la réalité telle que la vivent les acteurs eux-mêmes, en étant toujours «à la recherche du vivant » ${ }^{37}$, comme le dit Michel Maffesoli, que nous avons souhaité révéler la marge de liberté des récepteurs dans leur action. Le regard porté sur l'offre marchande des jeux en tant que produits de consommation de masse met en valeur une aliénation de l'individu consommateur. En considérant les motivations de l'acteur, nous l'appréhendons comme un récepteur actif.

\section{2-2 Un récepteur pas si passif}

Pour dépasser les aspects directement visibles dans le jeu de hasard, à savoir son aspect commercial et mercantile, le centre de recherches de Birmingham présente un intérêt majeur $^{38}$. Les études culturelles réhabilitent les pratiques populaires, en insistant sur le plaisir éprouvé par les récepteurs à leur usage. Richard Hoggart, lors de son étude ethnologique sur les pratiques culturelles des classes populaires britanniques, démontre que, contrairement aux

${ }^{36}$. HOGGART R., La Culture du pauvre, Paris, Minuit, 1970, p. 379.

${ }^{37}$. MAFFESOLI M., La Connaissance ordinaire. Précis de sociologie compréhensive, Paris, MéridiensKlincksieck, 1985.

${ }^{38}$. Créé en 1964, le centre de recherches de Birmingham, s'intéresse à l'apparition des formes culturelles liées à l'industrialisation et fait, dans les années 60-70, de la culture populaire, l'objet principal de ses études. Elle sera analysée en opposition avec les thèses critiques de l'Ecole de Francfort, en montrant que le récepteur est actif et doté de différents moyens de décodages des textes des médias (voir à ce propos HALL S., «Codage/décodage » in BEAUD P. (sous la direction de), Sociologie de la communication, Réseaux, CNET, 1997, pp. 61-71). 
discours communs, la presse spécialisée dans le traitement de la vie privée des célébrités n'a pas une grande influence dans les modes de pensée des lecteurs et qu'elle est surtout une source de plaisir et de distraction. Michel de Certeau, avec la "sociologie du quotidien », analyse les pratiques et les arts de faire comme autant de réponses aux théories du récepteur passif. Il revient surtout sur la thèse prônant la vulgarité de certains programmes : «Les protestations contre la vulgarisation/vulgarité des médias relèvent souvent d'une prétention pédagogique analogue ; portée à croire ses propres modèles culturels nécessaires au peuple en vue d'une éducation des esprits et d'une élévation des cœurs, l'élite émue par le "bas niveau" des canards ou de la télé postule toujours que le public est modelé par les produits qu'on lui impose. C'est là se méprendre de l'acte de "consommer" $»{ }^{39}$. Il conclut son chapitre consacré à la lecture par une mise au point : " Mais là où l'appareil scientifique (le nôtre) est porté à partager l'illusion des pouvoirs dont il est nécessairement solidaire, c'est-à-dire à supposer les foules transformées par les conquêtes et les victoires d'une production expansionniste, il est toujours bon de rappeler qu'il ne faut pas prendre les gens pour des idiots $"{ }^{40}$. Il présente les pratiques comme les instruments d'une signification donnée au monde social et qui permettent une réappropriation de l'espace social. Enfin, « en considérant que la sociologie de la vie quotidienne peut être une manière de comprendre [...] la socialité ${ }^{41}$, Michel Maffesoli parle de « quant-à-soi populaire » ${ }^{42}$ pour désigner les actes du quotidiens comme autant de résistances contre l'aliénation au sens où l'entend $\operatorname{Karl} \operatorname{Marx}^{43}$.

\section{2-3 L'activité ludique}

En nous appuyant précisément sur les résultats du travail d'enquête mené pendant deux ans, nous pouvons dire que, comme dans la conception grecque qui séparaient tukê, c'est-à-dire le hasard dans l'espace intentionnel humain et automaton, le hasard dans l'ordre de la nature, les enquêtés distinguent deux "hasards" selon les motifs de l'action et les choix qu'ils nécessitent. Plus généralement, les propos recueillis vont tous dans une même direction : en parlant de la pratique ludique en général et de leur expérience en particulier, les enquêtés décrivent une activité sociale et le hasard est absent des propos recueillis. Il a fallu introduire le sujet dans la discussion pour qu'il en soit question et il est remarquable que la totalité des enquêtés l'a reléguée au statut de facteur avec lequel il faut composer pour continuer l'action. De façon spontanée, c'est la finalité de la pratique qui est mise en avant de façon unanime. La motivation, c'est l'argent. Pour l'individu, il s'agit bien d'évacuer délibérément la notion d'aléa en s'appropriant l'action. Ainsi, le jeu est présenté comme une action que la personne contrôle, par le seul fait qu'elle se la soit appropriée. En décidant de jouer, en décidant de la nature du jeu, de la somme qu'il y engage ou encore du contexte, l'individu maîtrise son activité. Les questions du guide d'entretien concernant l'aléa dans la vie en général ont confirmé que les individus l'excluent de leurs actions. Ils peuvent l'admettre, quand ils ne le nient pas carrément, mais s'il survient malgré eux, l'action menée le jugulera de sorte que la finalité soit due au contrôle personnel. Pour parler en terme de stratégie d'action, il s'agit bien d'évacuer la source d'incertitude et, plus encore, de nier tout élément qui risquerait de perturber le bon fonctionnement de la « machine triviale » ${ }^{44}$ qu'est l'homme social. Dans le même ordre d'idée, nous constatons que les entretiens traduisent unanimement une ambivalence dont l'explication relève du manque de connaissance de la notion de « hasard»

\footnotetext{
39. DE CERTEAU M., L'Invention du quotidien. 1. Arts de faire, Paris, Gallimard, 1990, p. 240.

. Ibid., p. 255.

41. MAFFESOLI M., La Connaissance ordinaire, Paris, Klincksieck, 2007 (1995), p. 210.

42. MAFFESOLI M., Le Temps des tribus, Paris, Méridiens Klincksieck, 1988, p. 65.

43. MARX K., Le Capital. Livre Premier, Paris, Editions sociales, 1867.

${ }^{44}$. MORIN E., Introduction à la pensée complexe, Paris, Seuil, 2005, p. 109.
} 
qui produit de l'ambiguïté : l'individu ne peut se prononcer clairement sur un sujet qu'il ne connait pas. Nous notons d'ailleurs que les prises de positions vindicatives pour affirmer que le hasard existe ou pas, se voient le plus souvent contredites au cours de la conversation. Dans ce cadre, l'ambivalence de l'individu est la traduction de sa méconnaissance et engendre des réactions d'adaptation dénotant une instabilité. De plus, considérant l'activité et pas seulement l'action sociale, nous prenons en compte la dimension émotionnelle de l'individu que Michel Maffesoli invite à ne jamais omettre ${ }^{45}$. Pour Edgar Morin, il s'agit des sentiments ${ }^{46}$ et c'est la dimension qui permet de ne pas se borner à une vision de l'homme seulement dans sa dimension de sapiens. Suivant cette logique, nous ajoutons que la réaction d'adaptation que l'individu met en place face au hasard dépend, certes de sa méconnaissance, mais aussi de son inconstance originelle telle que Morin l'invoque pour parler des antagonismes de l'homme «biologico-social ${ }^{47}$. Si la réaction d'adaptation relève de l'ambivalence, c'est aussi parce que l'homme est inconstant. L'analyse de Patrick Roger ${ }^{48}$ à propos des joueurs de loto va également dans ce sens. À observer ce que l'auteur nomme «la psychologie des joueurs » ${ }^{49}$, nous voyons bien des exemples de comportements individuels qui ne relèvent pas de la rationalité, qu'il s'agisse de l'illusion de contrôle qui amène l'individu à penser qu'il possède des capacités influençant le jeu ou qu'il s'agisse de la surestimation de la rentabilité qui provient d'un biais mémoriel dû à ce que les psychologues appellent "l'heuristique de la disponibilité $\gg{ }^{50}$. C'est toute la problématique des théories de la rationalisation et de l'engagement, formulées notamment par Robert-Vincent Joulé et Jean-Léon Beauvois ${ }^{51}$, qui supposent que les acteurs sont des producteurs d'explications causales. Ainsi, tout individu serait à la recherche d'un équilibre entre ses pensées et ses actions. De fait, s'il vit une incohérence entre ses attitudes et d'autres ou bien s'il est en contradiction entre ses idées et certains de ses comportements contraints (qu'ils soient choisis ou extorqués), il va tenter de restaurer un lien de correspondance entre ces différents niveaux en générant une explication. Cette dernière permet de rétablir la dissonance cognitive, selon les termes de Léon Festinger $^{52}$. Il s'agit d'une activité de reconstruction cognitive, c'est-à-dire de consonance et d'attribution causale qui est qualifiée de « rationalisation ». Ainsi, la rationalisation est une forme d'activité idéologique qui, selon Jean-Léon Beauvois ${ }^{53}$, est d'autant plus importante, en nombre et en intensité, que les sujets ont le sentiment d'être libres alors que leur choix réel, dans les décisions qu'ils prennent, peut être objectivement restreint. La pratique de rationalisation engendrerait donc les opinions (notamment celle de liberté comme nous venons de le dire).

\section{Conclusion}

La perspective de l'action telle que nous l'avons envisagée consiste à aborder les motifs d'action comme des processus déterminants et agissants dans l'action, suivant le modèle épistémique systémique qui propose de considérer un instant unique dans lequel tout se passe, suivant l'idée que «non seulement la partie est dans le tout mais que le tout est dans la

\footnotetext{
45. MAFFESOLI M., Le Réenchantement du monde, Paris, La Table Ronde, 2007.

46. MORIN E., Ethique, Paris, Seuil, 2007.

47. MORIN E., Les Sept savoirs nécessaires à l'éducation du futur, Paris, Seuil, 2000.

48. ROGER P., Lotomania, Paris, Village Mondial, 2005.

49 . Ibid., pp. 145-168.

${ }^{50}$. Une heuristique est une règle de décision simplifiée. Pour des exemples d'heuristiques courantes, voir chapitre 3 in BROIHANNE M., Finance comportementale, Paris, Economica, 2004.

51 . JOULE R-V. et BEAUVOIS J-L., La Soumission librement consentie, Paris, PUF, 1998.

${ }^{52}$. FESTINGUER L., RIECKEN H. et SCHACHTER S., L'Echec d'une prophétie. Psychologie sociale d'un groupe de fidèles qui prédisaient la fin du monde, Paris, PUF, 1993.

53. BEAUVOIS J-L., Traité de servitude libérale, Paris, Dunod, 1994.
} 
partie $»^{54}$, contrairement aux deux paradigmes déterministe et individualiste méthodologique qui sont traditionnellement présentés comme opposés l'un à l'autre mais qui, concernant le concept d'action, se réunissent sous un même modèle épistémique que l'on peut qualifier de linéaire. L'opposition entre les deux schémas repose alors sur le degré d'engagement des processus dans l'action. Pour les premiers, ce sont les processus sociaux et sociétaux mis en œuvre à un instant $\mathrm{A}$ («monde des prédispositions ») qui auraient le plus d'influence sur l'instant B («monde des choix, des dispositions et des actions »). En revanche, pour les seconds, ce sont des processus «dynamiques » (domaines propres à l'acteur autonome : c'est par exemple l'intuition) de l'instant A qui détermineraient le plus puissamment l'instant B ( "monde de la rationalité limitée »). La force des motifs d'action diverge donc, donnant plus ou moins de qualité déterminante à tel ou tel processus, mais le modèle reste toujours linéaire : un instant A tend vers un instant B. Pour nous, le principe hologrammatique de la pensée complexe présente l'intérêt, notamment, d'introduire des notions telles que l'aléa, l'inattendu, l'incontrôlable, en un mot le désordre, en lui accordant une place à part entière. Il ne s'agit plus de voir l'action seulement comme une décision, un choix déterminé par des facteurs bien identifiables et identifiés, mais aussi comme un pari. Or, nous dit Edgar Morin, « dans la notion de pari, il y a la conscience du risque et de l'incertitude » ${ }^{55}$. Ainsi, l'action est stratégie et, face au hasard, elle ne se borne pas à lutter contre dans une recherche d'information, elle essaie aussi de l'utiliser. Mais l'action aussi «entre dans un univers d'interactions et c'est finalement l'environnement qui s'en saisit ${ }^{56}$. C'est pourquoi, elle échappe à nos intentions et à des causes toujours parfaitement identifiables puisque notre monde environnant recèle d'éléments qui dépassent notre entendement, notre connaissance, ce que même notre esprit, tout fécond soit-il, ne peut concevoir. En effet, la vie sociale exige que nous nous conduisions comme des machines triviales selon la définition qu'en donne Edgar Morin : " est triviale une machine dont, si vous connaissez tous les inputs, vous connaissez tous les outputs $»^{57}$, mais le monde qui nous entoure, lui, impose de prendre en compte tout ce qui concerne le surgissement du nouveau, qui est non trivial et ne peut être prédit à l'avance. Ce qui nous amène à considérer que l'action ne peut être réduite au concept d'activité sociale telle que la définit Max Weber, c'est-à-dire dans sa dimension de comportement orienté par autrui, ni aux seuls déterminants qui, selon lui, orientent l'action «a) de façon rationnelle en finalité [zweckrational], par des expectations du comportement des objets du monde extérieur ou de celui d'autres hommes, en exploitant ces expectations comme "conditions" ou comme "moyen" pour parvenir rationnellement aux fins propres, mûrement réfléchies, qu'on veut atteindre ; b) de façon rationnelle en valeur [wertrational], par la croyance en la valeur intrinsèque inconditionnelle - d'ordre éthique, esthétique, religieux ou autre - d'un comportement déterminé qui vaut pour lui-même et indépendamment de son résultat; c) de façon affectuelle [affektuel], et particulièrement émotionnelle, par des passions et des sentiments actuels; d) de façon traditionnelle [traditional], par coutume invétérée $»^{58}$. La dimension rationnelle est évidente et on peut voir dans les dimensions « affectuelle » et « traditionnelle » que l'auteur reconnaît et intègre l'idée de l'irrationnel au processus de l'activité. Cependant, Edgar Morin rappelle l'enseignement de Pierre Auger qui a fait remarquer qu'on ne peut « se borner au diptyque rationnel-irrationnel. Il faut ajouter l'a-rationnel : l'être et l'existence ne sont ni absurdes ni rationnels ; ils sont ${ }^{59}$.

\footnotetext{
${ }^{54}$. MORIN E., Science avec conscience, Paris, Fayard, 1982, p. 168.

55 . Ibid., p. 105.

${ }^{56}$. Ibid., p. 107.

${ }^{57}$. MORIN E., Introduction à la pensée complexe, Paris, Seuil, 2005, p. 109.

58. WEBER M., «Les Catégories de la sociologie», Economie et société, Paris, PLON, 1995 (1922), Tome 1, p. 55.

59. MORIN E., Science avec conscience, Paris, Fayard, 1982, p. 155.
} 
Et encore, il faut « reconnaître également le sur-rationnel (Bachelard). Sans doute [...] il y a des phénomènes à la fois irrationnels, rationnels, $a$-rationnels, sur-rationnels » ${ }^{60}$ En d'autres termes, Max Weber reconnaîtrait la notion d'ordre à travers les deux premiers types d'action qu'il définit et celle de désordre via les deux dernières ; mais, de par sa typologie même, il séparerait peut-être les deux faces d'un même phénomène. Il convient donc de rétablir le dialogue entre ordre et désordre et, pour ce faire, Edgar Morin propose un tétragramme entre ordre, désordre, interaction et organisation, qui «nous permet de concevoir que l'ordre de l'univers s'autoproduit en même temps que s'autoproduit cet univers, à partir des interactions physiques qui produisent de l'organisation mais aussi du désordre $»^{61}$. Finalement, nous dit l'auteur, "l'action suppose la complexité, c'est-à-dire aléa, hasard, initiative, décision, conscience des dérives et des transformations » ${ }^{62}$ mais, comme le montre le tétragramme, « il n'y aura jamais un maître mot - une formule maîtresse - qui commandera l'univers ${ }^{63}$. Il n'est donc pas question pour nous de renier tout déterminisme dans l'action en cherchant une autre vérité; la pensée complexe représente plutôt une façon de penser, une certaine gymnastique d'esprit à laquelle nous avons tenté de nous livrer le plus possible malgré les barrières de notre pensée « dualisante ».

Finalement, en traitant des motifs d'action du joueur de jeux de hasard, nous avons mis en valeur le rapport entre la rationalité et l'irrationalité qui s'exprime dans l'activité sociale. L'objet du jeu de hasard pur a mis en valeur trois caractéristiques principale : le ludisme, le hasard et l'argent. Cependant, en cherchant à comprendre la pratique de l'action, il s'avère que l'attention se focalise sur le rapport au hasard en général. Pour en traiter, l'activité ludique semble intéressante et demanderait à être approfondie, notamment par une étude plus étendue des processus déterminants et actifs dans l'action ainsi que dans la personnalité du joueur qui pourrait être exprimée en termes de « formes identitaires ». Ces derniers seraient définis selon différents niveaux d'engagement et s'ajusteraient selon l'agencement des processus (plus ou moins déterminants). Le terme de "forme» rendrait compte de la nature mouvante et malléable de cibles identifiées, certes, mais qui ne sont que potentielles et sans frontière ; à l'instar des « identités emboîtés » d'Edmond Bernus ${ }^{64}$ pour qui les espaces s'emboîtent au fil des relations tissées. En même temps, l'individu qui entre en contact avec le nouvel espace développe un sentiment d'appartenance à ce territoire. Si parler de territoire et de sentiment d'appartenance semble par trop renvoyer à la notion de communauté, l'idée qu'un même processus sera plus ou moins déterminant selon le contexte qui entoure l'acteur rejoint celle des processus telle que nous les avons évoqués. L'idée que nous formulons ici, à titre d'ouverture, consiste, d'une manière générale, à considérer un approfondissement de l'étude de la ludicité, suivant la conception de Jean Duvignaud ${ }^{65}$ selon qui il existe en l'homme une part de ludique inflexible à toutes les structures (sociale, parentale...), à toutes les activités, même, des sociétés qui tentent de la récupérer (ou de la sacraliser).

Pour ce faire, on pourrait étudier plusieurs pratiques ludiques différentes, mais surtout le jeu dans ses multiples acceptions (jeu politique et jeu sentimental par exemple), afin d'approcher l'attitude ludique telle que la considère Jean Duvignaud : libre et gratuite.

\footnotetext{
${ }^{60}$. Ibid., pp. 155-156.

61 . Ibid., p. 189.

62. MORIN E., Introduction à la pensée complexe, Paris, Seuil, 2005, p. 107.

63. Op. Cit., p. 191

${ }^{64}$ BERNUS E., 1995, «Perception du temps et de l'espace par les Touaregs nomades sahéliens », in CLAVAL P., Ethnogéographie, Paris, l'Harmattan.

${ }^{65}$. DUVIGNAUD J., Le Jeu du jeu, Paris, Balland, 1980.
} 


\section{Bibliographie}

BACHELARD G., Le Matérialisme rationnel, Paris, PUF, 2000.

BAUDRILLARD J., Le Système des objets, Paris, Denoël-Gonthier, 1968.

BAUDRILLARD J., L'Echange symbolique et la mort, Paris, Gallimard, 1976.

BEAUVOIS J-L., Traité de servitude libérale, Paris, Dunod, 1994.

BERNUS E., 1995, "Perception du temps et de l'espace par les Touaregs nomades sahéliens ", in CLAVAL P., Ethnogéographie, Paris, l'Harmattan.

BROIHANNE M., Finance comportementale, Paris, Economica, 2004.

CASTORIADIS C., L'Institution imaginaire de la société, Paris, Seuil, 1999.

DE CERTEAU M., L'Invention du quotidien. 1. Arts de faire, Paris, Gallimard, 1990.

DOSTOÏEVSKI F., Le Joueur, Paris, Gallimard, 1977 (1865).

DURKHEIM E., Les Formes élémentaires de la vie religieuse, Paris, Librairie générale française, 1991 (1912).

DUVIGNAUD J., LeJjeu du jeu, Paris, Balland, 1980.

FESTINGUER L., RIECKEN H. et SCHACHTER S., L'Echec d'une prophétie. Psychologie sociale d'un groupe de fidèles qui prédisaient la fin du monde, Paris, PUF, 1993.

HOGGART R., La Culture du pauvre, Paris, Minuit, 1970.

JOULE R-V. et BEAUVOIS J-L., La Soumission librement consentie, Paris, PUF, 1998.

LEGROS P., MONNEYRON F., RENARD J-B. et TACUSSEL P., Sociologie de l'imaginaire, Paris, Armand Colin, 2006.

MAFFESOLI M., La Connaissance ordinaire. Précis de sociologie compréhensive, Paris, Méridiens-Klincksieck, 1985.

MAFFESOLI M., Le Temps des tribus, Paris, Méridiens Klincksieck, 1988.

MAFFESOLI M., La Connaissance ordinaire, Paris, Klincksieck, 2007 (1995).

MAFFESOLI M., Le Réenchantement du monde, Paris, La Table Ronde, 2007.

MARX K., Le Capital. Livre Premier, Paris, Editions sociales, 1867.

MORIN E., L'Esprit du temps. I Névrose, Paris, Grasset, 1962.

MORIN E., Science avec conscience, Paris, Fayard, 1982.

MORIN E., La Complexité humaine, Paris, Flammarion, 1994.

MORIN E., Les Sept savoirs nécessaires à l'éducation du futur, Paris, Seuil, 2000.

MORIN E., Introduction à la pensée complexe, Paris, Seuil, 2005.

MORIN E., Ethique, Paris, Seuil, 2007.

RIVIERE C., «Pour une théorie du quotidien ritualisé », Ethnologie française, n²6, Paris, Armand Colin, 1996.

ROGER P., Lotomania, Paris, Village Mondial, 2005.

WEBER M., «Les Catégories de la sociologie », Economie et société, Paris, PLON, 1995 (1922), Tome 1.

WOLTON D., Eloge du grand public. Une théorie critique de la télévision, Paris, Flammarion, 1990.

Dictionnaire Le Robert, Paris, Nathan, 1991. 